\title{
Composantes de la croissance en hauteur chez le Pin maritime (Pinus pinaster Ait.)
}

\author{
A. KREMER et G. ROUSSEL \\ I.N.R.A., Laboratoire d'Amélioration des Arbres forestiers \\ Pierroton, F 33610 Cestas
}

\begin{abstract}
Résumé
Le dénombrement des entre-nouds présents sur une pousse annuelle, le suivi de leur élongation, le suivi de la croissance annuelle. ont permis de décrire les principales étapes de l'élaboration d'une pousse annuelle en faisant également appel à l'étude morphogénétique réalisée par Morisset (1966). Autant au niveau de l'initiation qu'à celui de l'élongation, il y a un décalage dans la chronologie des opérations entre clones polycycliques et clones monocycliques. Les méristèmes apicaux continuent à fonctionner durant l'hiver chez les premiers et initient durant cette époque la seconde pousse. Il y a un allongement précoce du bourgeon terminal des monocycliques durant l'été et l'automne précédant l'année d'élongation proprement dite.

Au total, les arbres polycycliques «fabriquent» plus daiguilles de branches, et de cônes et les arbres monocycliques ont un accroissement total annuel plus important que l'accroissement primaire des polycycliques, qui est laccroissement "morphogénétiquement » homologue. Ce supplément est dû en partie à l'allongement précoce du bourgeon mais aussi à un allongement supérieur des entre-noeuds en période de croissance maximale, au printemps.

Par ailleurs, à l'intérieur de la population monocyclique, laccroissement total est surtout expliqué, par l'élongation des entre-nœuds aux différents niveaux. Dans la population polycyclique, e'est le nombre d'entre-neuds et leur élongation au sommet qui explique au mieux l'accroissement correspondant au premier cycle morphogénétique.
\end{abstract}

Mots clés: Morphogénèse, croissance en hauteur, initiation des euphylles, élongation des entre-nouds, polycyclisme, Pinus pinaster.

\section{I. - Introduction}

La pousse totale annuelle de la plupart des conifères peut être décomposée en plusieurs facteurs : nombre de pousses qui se développent chaque année et à l'intérieur de chacune d'entre elles, nombre d'entre-nœuds présents et allongement de ces entrenœuds. Cette décomposition correspond d'ailleurs à la succession des phases du développement végétatif qui aboutit à la constitution d'une pousse : initiation des pousses, des entre-nœuds et allongement de ces structures initiées. C'est à partir de cette 
décomposition que les travaux portant sur la croissance en hauteur ont récemment été poursuivis (CANnell et al., 1976 ; CanNell, 1978 ; Lanner, 1976 ; LanNer, 1978 ; Pollard \& Logan, 1976 ; Pollard \& Logan, 1977). Et c'est principalement la première phase de la morphogénèse de la pousse qui a été abordée. En effet, dans de nombreux exemples (Pinus contorta, VAN DEN BERg \& LANNER, 1971, et Picea sitchensis, CANNELl et al., 1976), il existait une corrélation très étroite entre nombre de brachyblastes présents sur la pousse annuelle et la longueur de la pousse, à tel point que l'importance de l'élongation elle-même n'était plus que mineure.

L'accroissement annuel du pin maritime est caractérisé par la présence d'un ou de deux cycles morphogénétiques (Debazac, 1963). Un cycle morphogénétique correspond à une succession donnée d'éléments : écailles, brachyblastes, auxiblastes et cônelets. Les pins polycycliques ont généralement un accroissement annuel plus important que les pins monocycliques, alors que laccroissement de la pousse primaire (correspondant au premier cycle morphogénétique) des premiers est toujours inférieur à l'accroissement total des seconds (MAUGE et al., 1976 ; KREMER, 1981).

Dès lors, le polycyclisme appelait plusieurs questions eu égard aux composantes de la croissance en hauteur.

- Comment se différencient les arbres monocycliques et polycycliques au niveau de l'initiation des éléments de l'accroissement annuel et de l'élongation des entre-nœuds?

- Par quelle composante peut être expliqué le supplément de vigueur obtenu par les polycycliques?

C'est à partir de ces questions et de leurs réponses que la présente contribution se propose de dégager des conclusions au niveau de la sélection et de l'amélioration génétique du pin maritime.

\section{II. - Matếriel et méthodes}

Le matériel végétal sur lequel ont porté les mesures est constitué de clones monocycliques et polycycliques. Ces clones ont pu être identifiés à partir de mesures antérieures du nombre de pousses secondaires exprimées sur $\mathrm{n}$ années dans différents dispositifs décrits dans une étude plus complète portant sur le polycyclisme (Kremer, 1981). Certains ont été choisis à partir de leur valeur en test clonal, d'autres à partir de leur valeur en test de descendances de demi-frères. On peut considérer que la sélection des clones dans les deux dispositifs a abouti aux mêmes résultats dans la mesure où l'héritabilité au sens strict du caractère précisé plus haut était de l'ordre de 0,44 (KREMER, 1981). C'est de manière très empirique que l'identification de clones polycycliques et monocycliques a été faite : en tronquant la courbe de distribution du nombre de pousses secondaires aux deux extrémités.

Tous les clones font partie de la population landaise et ont été mesurés dans le parc à clones des arbres + situé en parcelle J du Domaine de l'Hermitage. Les clones ont été greffés en 1964. Les mesures ont été de deux types. 


\section{1. - Courbes de croissance annuelle}

Le niveau acquis par la pousse annuelle a été relevé à 19 reprises entre le 6 mars et le $1^{\text {er }}$ octobre 1979 , sur 3 clones monocycliques et 3 clones polycycliques, chaque clone étant représenté par deux copies. Chaque relevé correspond donc à :

- l'ensemble pousse primaire + bourgeon en formation pour les monocycliques (voir figure $1 \mathrm{~A}$ ) ;

- l'ensemble pousse primaire + pousse secondaire + bourgeon en formation pour les clones polycycliques (voir figure $1 \mathrm{~B}$ ).

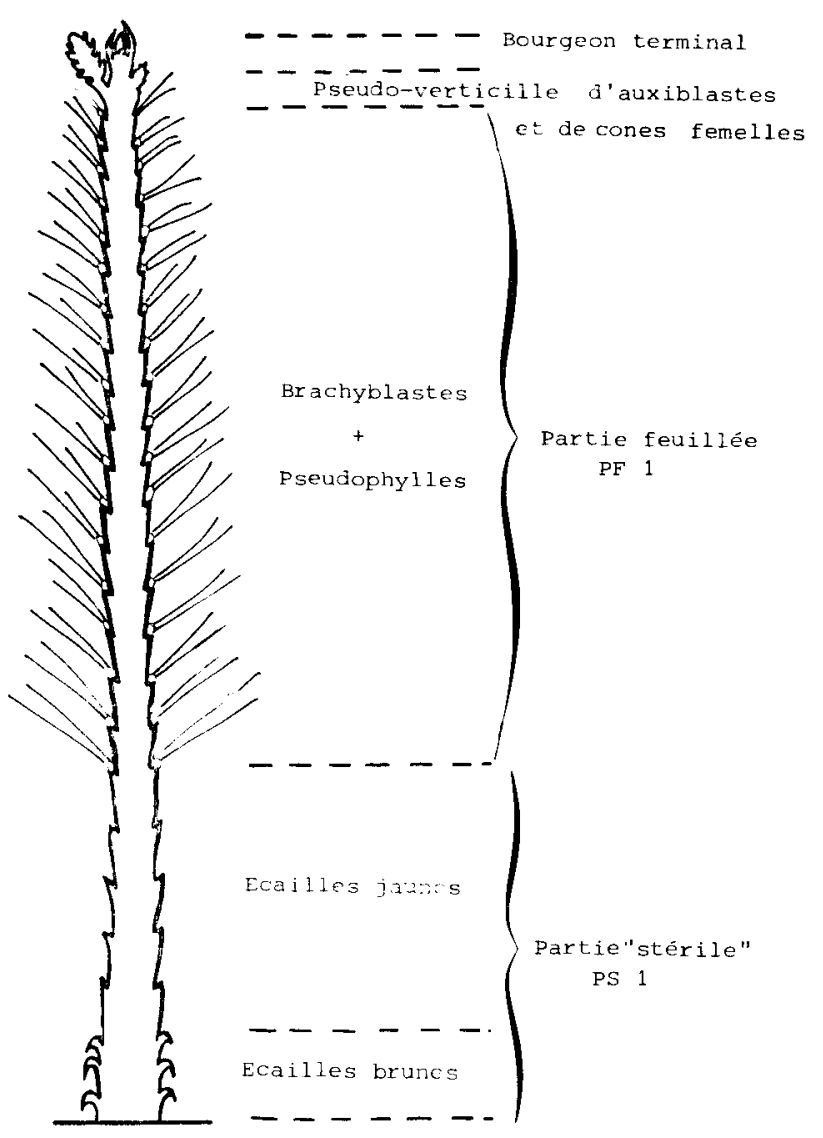

FIG. 1 A

Description du cycle morphogénétique des clones monocycliques

Description of the morphogenetic cycle of monocyclic clones

Dès que les auxiblastes se sont différenciés en rameau au niveau du pseudoverticille intermédiaire, il a été possible de noter l'élongation de la partie supérieure à ce verticille c'est-à-dire :

— du bourgeon terminal pour les clones monocycliques; 

cycliques.

- de l'ensemble pousse secondaire + bourgeon terminal pour les clones poly-

Enfin, du 17 septembre 1979 au 6 février 1980, seule l'élongation du bourgeon terminal a été suivie à 7 reprises.

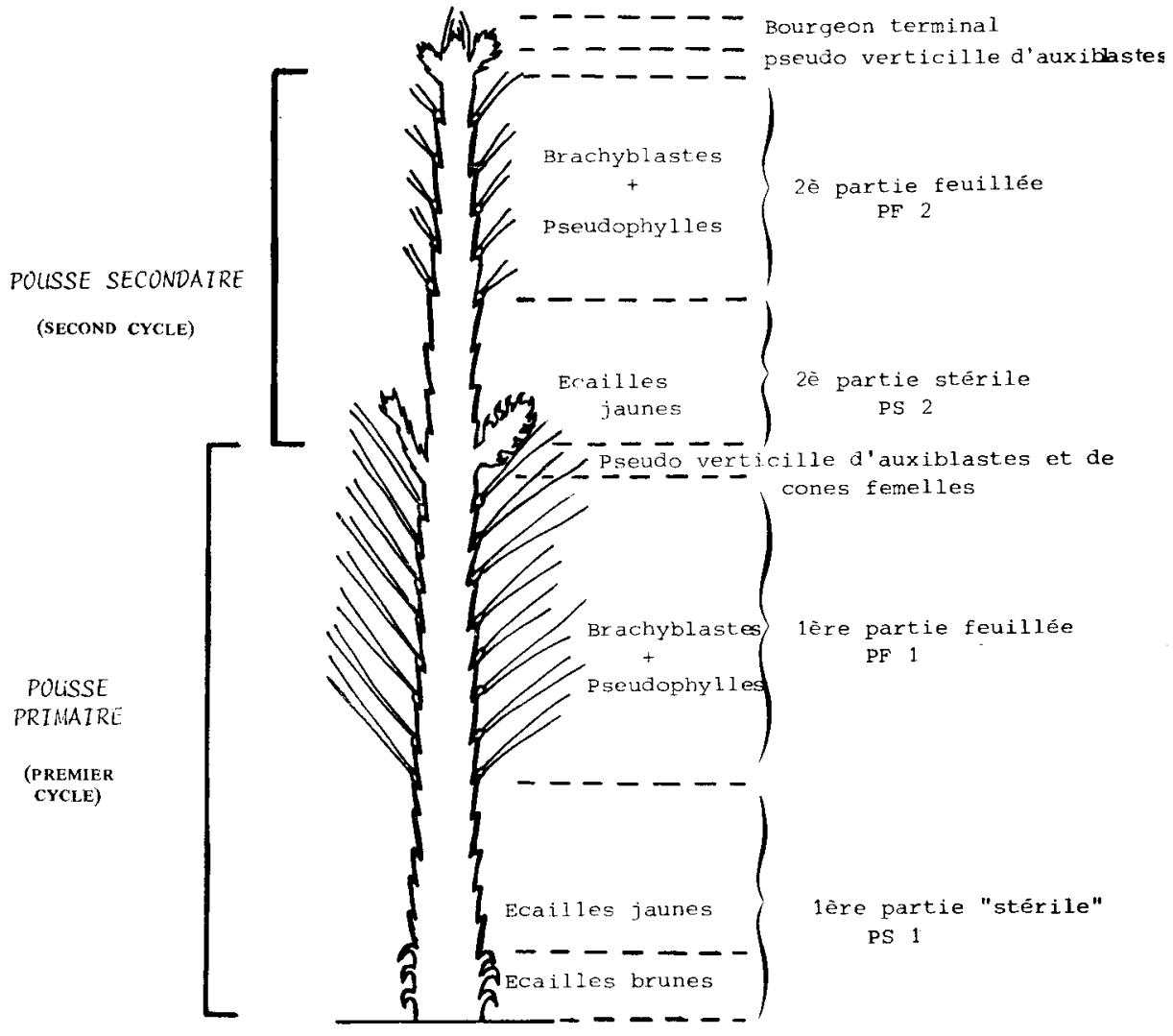

FIG. $1 \mathrm{~B}$

Description des cycles morphogénétiques des clones polycycliques

Description of the morphogenetic cycles of polycyclic clones

\section{2. - Décomposition de l'accroissement annuel}

La pousse primaire et la pousse secondaire pour les polycycliques, la pousse totale pour les monocycliques sont constituées d'un ensemble d'entre-nœuds qui, à leur extrémité, portent une bractée écailleuse, appelée euphylle par DEBAZAC (1963) et «cataphyll» par les anglo-saxons (CANNELL, 1976) (voir figure 2). L'ensemble entre-nœud et euphylle est appelé «stem unit» par DoAK (1935). A l'aisselle des 
euphylles se trouvent des points végétatifs dont le développement peut aboutir à des structures différentes (voir figure $1 \mathrm{~A}$ et $1 \mathrm{~B}$ ) :

- ces points végétatifs ne se développent pas du tout à la base de la pousse. Pour cette raison, ces euphylles sont souvent appelées écailles «stériles» et la longueur sur laquelle elles se situent partie «stérile»;

— après la partie «stérile » les points végétatifs se développent en rameaux courts ou brachyblastes qui porteront les aiguilles. D'où le nom de partie feuillée ;

- enfin, au sommet de la pousse, ils aboutiront à la formation d'auxiblastes et de cônelets.

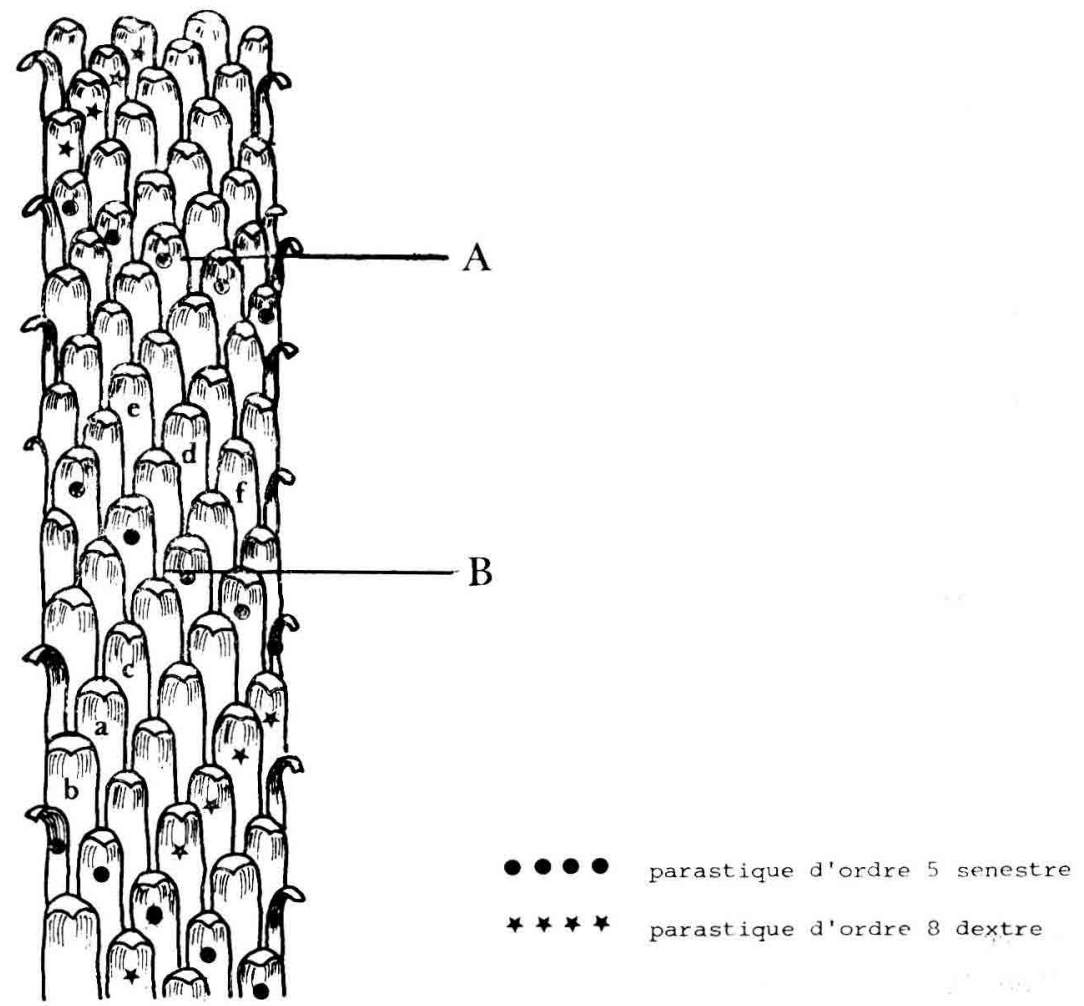

FIG. 2

Description d'une partie de la pousse annuelle - Arrangements phyllotaxiques des euphylles Description of a segment of the annual shoot-Phyllotactic arrangements of the cataphylls

Les points A et $B$ sont les intersections d'un parastique d'ordre 5 avec la verticale passant. au niveau du tronc et sont séparés de $5 \mathrm{n}$ entre-nœuds où $\mathrm{n}$ est le nombre d'entre-nœuds sur une révolution du parastique donné. Sur ce schéma ne sont représentées que les euphylles ou cataphylles : les structures axillaires (brachyblastes, auxiblastes ou conelets) n'ont pas été dessinées. 
L'ensemble des euphylles et de leurs ébauches axillaires se trouvent dans le bourgeon au début de la saison de végétation. Après laallongement télescopique de la tige embryonnaire, à chaque euphylle correspondra un entre-nœud.

Dans tout ce qui suit, le terme «article» désignera l'unité morphogénétique correspondant à l'euphylle (écaille) quel que soit l'organe différencié à partir du point végétatif qui se trouve à son aisselle (brachyblaste, auxiblaste, cônelet). Le terme article s’applique également aux écailles stériles. L'entre-nœud désigne l'espace séparant deux articles successifs, après élongation de l'ensemble.

Par convention, et pour lever toute ambiguité, dans tout ce qui suit, on utilisera les définitions suivantes ayant trait aux différents termes de croissance eu égard aux organes et aux structures auxquels ils s'adressent :

- laccroissement désigne la longueur de la pousse ou d'une partie de la pousse (cycle, partie «stérile» ou feuillée, bourgeon) quand celle-ci a atteint sa valeur maximale ;

- l'allongement désigne la longueur finale atteinte par un entre-noud ;

- l'élongation s'applique indifféremment à la pousse, à une partie de la pousse, à un entre-nœud, pour désigner la croissance en cours de chacune de ces structures. s'adresse.

Le terme élongation sera donc complété par le nom de la structure à laquelle il

Sur 19 clones monocycliques et 15 clones polycycliques, l'accroissement total de l'année 1979 a ainsi été décomposé en plusieurs parties, dont la longueur a été mesurée.

- Pour les clones polycycliques (voir figure 1 B)

- partie «stérile» de la pousse primaire (PS 1);

- partie feuillée de la pousse primaire (PF 1);

- partie «stérile» de la pousse secondaire (PS 2);

- partie feuillée de la pousse secondaire (PF 2).

- Pour les clones monocycliques (voir figure 1 A)

- partie «stérile» (PS 1);

- partie feuillée (PF 1).

\subsection{1. - Allongement moyen des entre-næuds}

A la fin de la saison de végétation, la longueur moyenne des entre-nœuds a été mesurée à plusieurs niveaux, qu'il s’agisse de clones monocycliques ou polycycliques :

- Pour les clones polycycliques

- à 3 niveaux sur la partie feuillée de la pousse primaire (PF 1): à la base au milieu et au sommet ;

- au milieu de la pousse secondaire (PF 2).

- Pour les clones monocycliques

- à trois niveaux sur la partie feuillée de la pousse (PF 1) : à la base, au milieu et au sommet.

En se référant aux arrangements phyllotaxiques, il est aisé de mesurer ces allon- 
gements. Le principe est de rapporter la distance séparant deux repères sur la tige au nombre d'entre-nouds les séparant. Les arrangements phyllotaxiques permettent de dénombrer les entre-nœuds séparant deux repères pourvu que ces derniers se trouvent, par exemple, sur des contacts parastiques bien définis. La définition de ce dernier terme suit. Si l'on observe la disposition des articles sur une tige (figure 2) des contacts de même nature unissent l'écaille a à l'écaille b et l'écaille a à l'écaille c. Ainsi les articles unis par juxtaposition de cette manière forment une ligne fictive, une spirale qui s'enroule autour du tronc et qui est appelée contact parastique ou parastique (CAMEFORT, 1956). Le même raisonnement peut être fait pour les écailles e, d, f, dont le sens d'enroulement du parastique les reliant, est différent du précédent. Si l’on dénombre les parastiques dextres et senestres, on obtient généralement dans le cas du pin maritime, des nombres faisant partie de la série de Fibonacci, dans le cas présent sur la figure 2,5 et 8 , mais dans le cas le plus général 3 et 5 . Ces chiffres font partie de la série principale : $1,2,3,5,8,13,21$, etc.

$A$ un instant $t$ de l'élongation de la tige, on ne distingue que deux parastiques d'ordre consécutif et dont le sens est opposé (CANNell \& Bowler, 1978). Dans la tige embryonnaire, ce sont les parastiques d'ordre élevé (8 et 13), qui sont visibles. Quand la tige est allongée ce sont les parastiques d'ordre plus faible (3 et 5 ).

A titre indicatif, sur les 68 arbres mesurés :

57 observaient la série $1,2,3,5,8 \ldots$

5 observaient la série $2,4,6,10 \ldots$

4 observaient la série $3,4,7,11 \ldots$

2 observaient la série $3,6,9,15 \ldots$

Pour mesurer l'allongement entre les articles, on choisit arbitrairement un parastique d'ordre $\mathrm{k}$ donné et on compte le nombre $\mathrm{n}$ d'articles au cours d'une révolution autour de la tige. Deux points $A$ et $B$ situés sur les intersections d'une génératrice avec le parastique choisi sont donc séparés de $\mathrm{k} n$ articles. L'allongement moyen des entre-nouds correspondant est donc $\mathrm{AB} / \mathrm{kn}$ (figure 2).

\subsection{2. - Dénombrement des articles}

- Sur les clones monocycliques, le nombre total de brachyblastes, de branches et de cônes a été noté par comptage direct.

- Sur les clones polycycliques, les mêmes dénombrements ont été faits sur la pousse primaire. Le nombre de branches sur le second pseudo-verticille $\left(^{*}\right)$ a également été noté. Il n'y a jamais eu de fructification femelle à ce niveau. Enfin, les brachyblastes sur la seconde pousse ont été dénombrés. Le nombre d'écailles "stériles》 sur la pousse secondaire a été calculé en comptant le nombre d'écailles sur un parastique et en le multipliant par le nombre de parastiques dans le même sens.

L'ensemble des articles présents sur une tige a été dénombré mis à part les écailles stériles de la pousse primaire. En effet les écailles brunes sont très imbriquées les unes dans les autres et rendent difficile leur dénombrement.

(*) Les auxiblastes sont situés sur les parastiques et suivent la même disposition que les brachyblastes. Développés à l'extrémité d'entre-nœuds différents, ils se trouvent à des niveaux différents. Le terme verticille ne peut donc être utilisé. Nous proposons le terme "pseudoverticille » utilisé par DEBAZAC. 


\section{III. - Résultats}

\section{1. - Courbes de croissance}

Les principaux résultats se dégageant de l'étude des courbes de croissance des clones polycycliques et monocycliques peuvent se résumer en plusieurs points :

- Il n'apparaît pas de différence entre polycycliques et monocycliques quant à la durée d'élongation (voir fig. 3 et 4). De la même manière les dates de début et d'arrêt de croissance coïncident. L'essentiel de l'accroissement annuel est réalisé entre la mi-avril et la mi-juin.

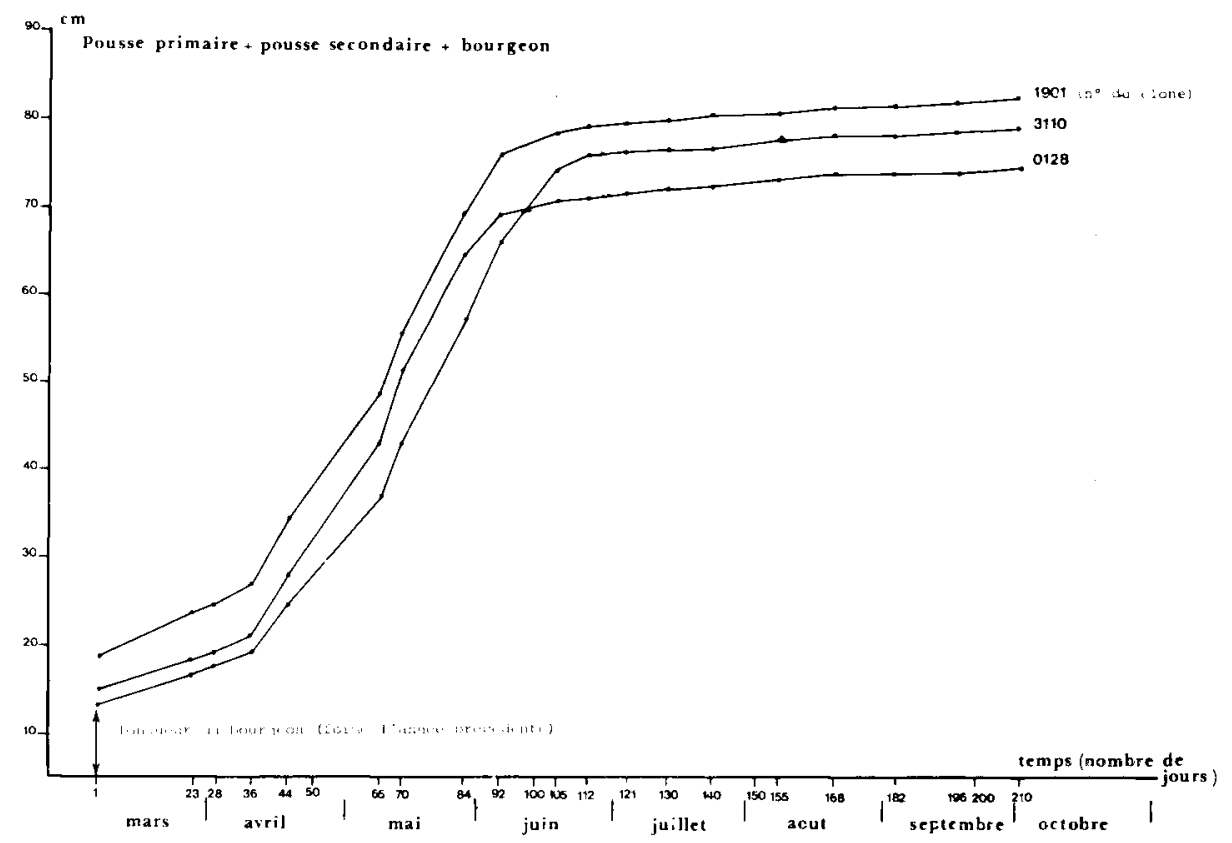

FIG. 3

Courbes de croissance des clones polycycliques

(Pousse primaire + pousse secondaire + bourgeon)

Growth curves of polycyclic clones (first shoot + second shoot + bud)

- Pour les clones polycycliques, la pousse secondaire s'allonge entre la mi-mai et la mi-juin (voir fig. 5). L'élongation de cette dernière se réalise simultanément avec celle des derniers entre-nœuds de la pousse primaire. Il n'y a pas de rupture dans la courbe de croissance au niveau du départ de la «pousse secondaire». 


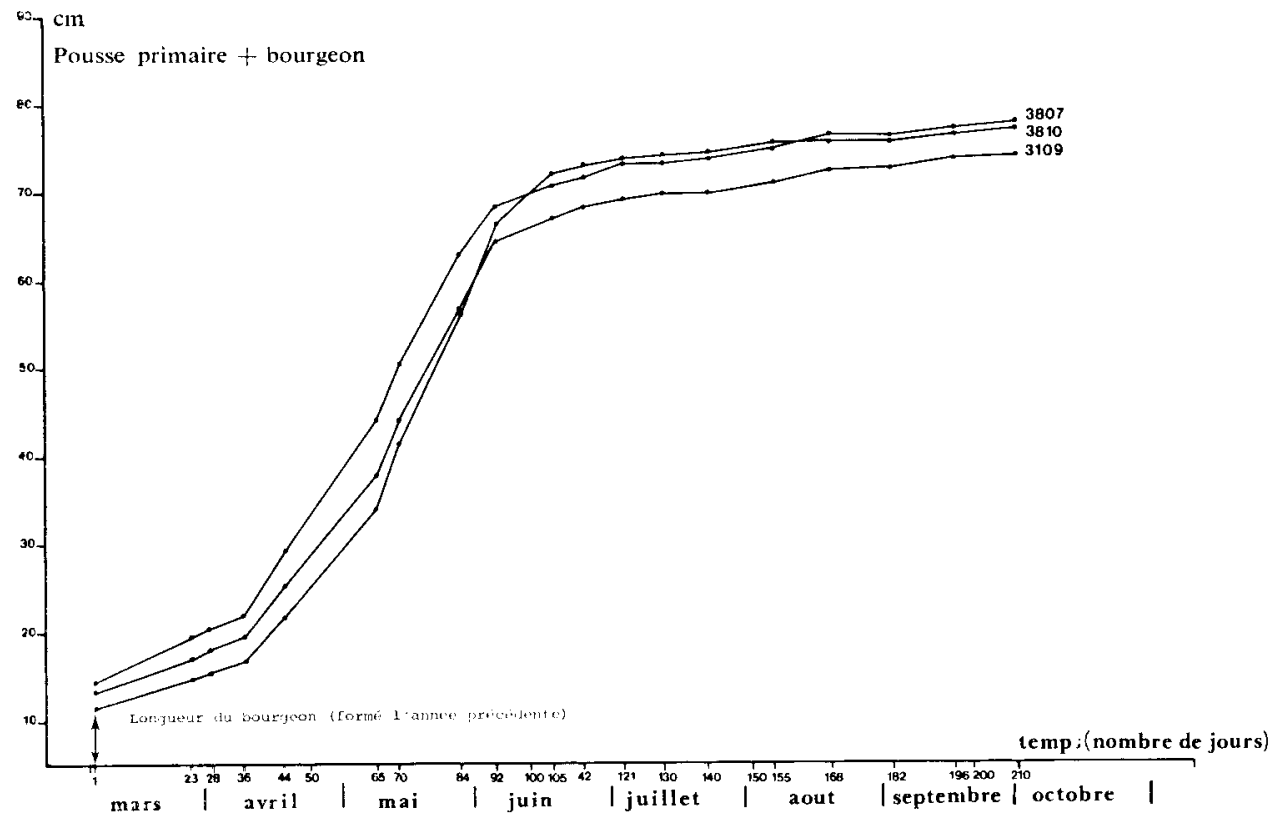

FIG. 4

Courbes de croissance des clones monocycliques (pousse primaire + bourgeon) Growth curves of monocyclic clones first (shoot + bud)

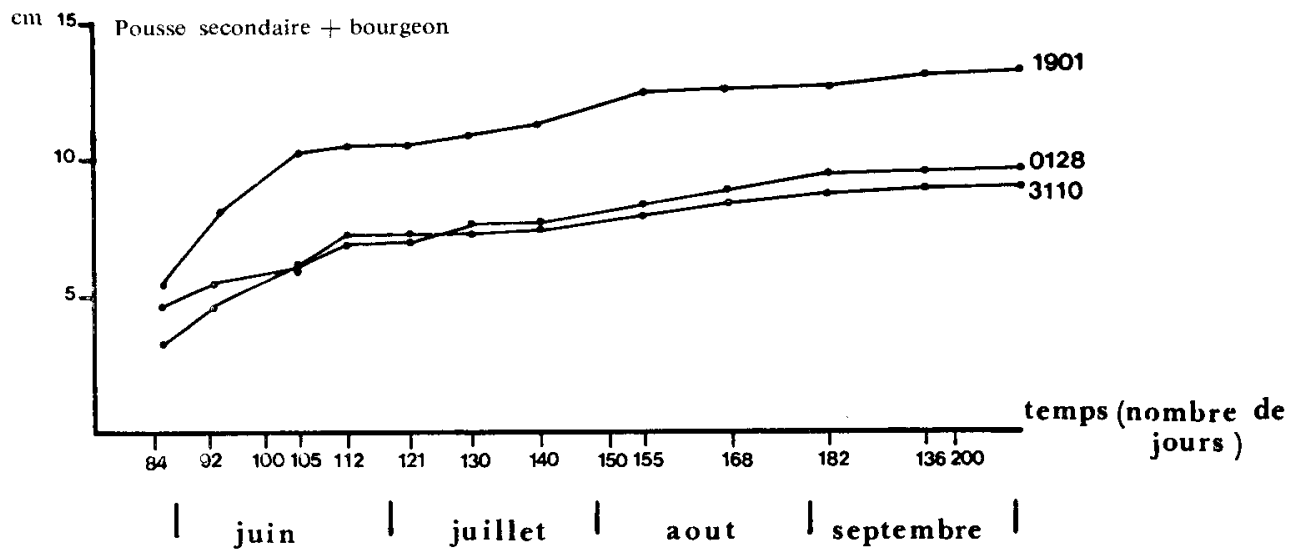

FIG. 5

Courbes de croissance de l'ensemble pousse secondaire + bourgeon de clones polycycliques Growth curves of monocyclic clones (first shoot + bud) 
- Les bourgeons des clones monocycliques s'allongent durant la période estivale (de juin à septembre) (cf. fig. 6). Ceci est très certainement dû à une élongation précoce des entre-nœuds correspondant aux écailles «stériles» de la pousse primaire de l'année suivante. La croissance des bourgeons s'arrête vers le mois de novembre (voir fig. 7). Une très légère évolution est encore visible durant l'hiver.

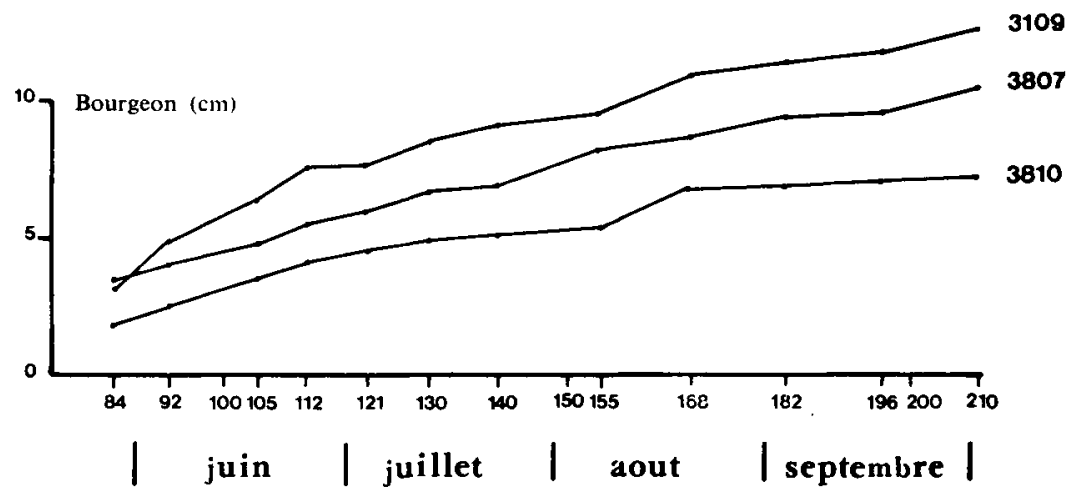

FIG. 6

Courbes de croissance du bourgeon de clones monocycliques Growth curves of the terminal bud of monocyclic clones

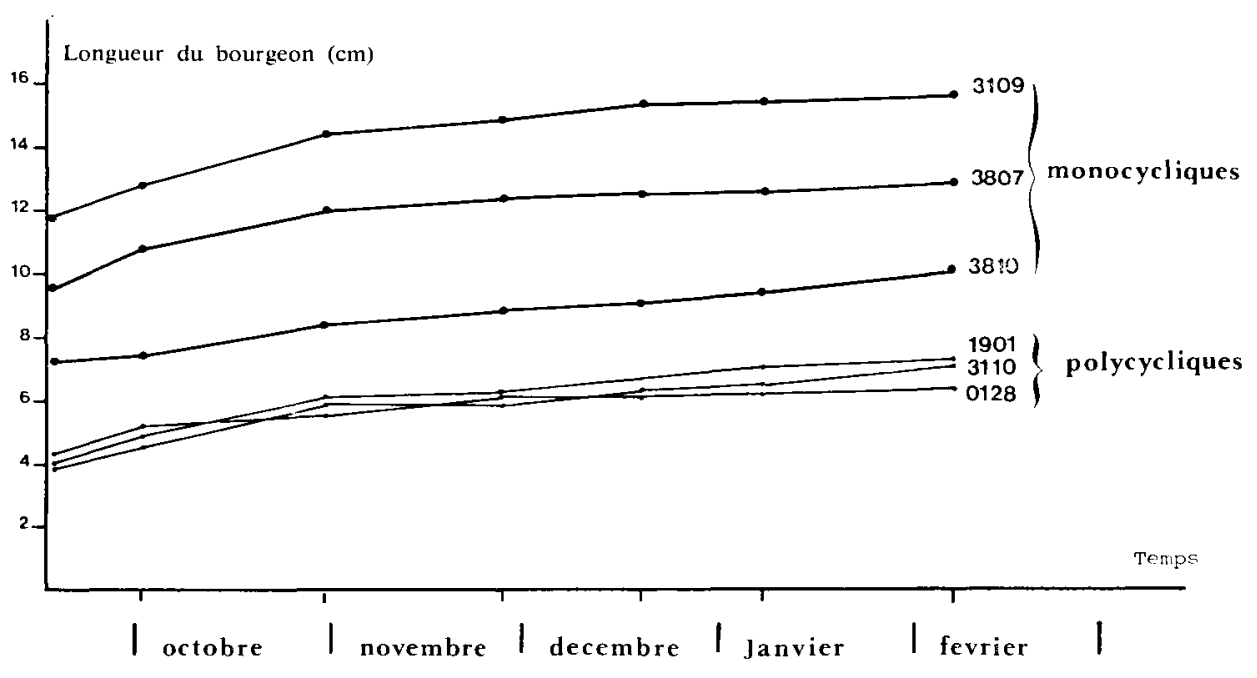

FIG. 7

Croissance hivernale des bourgeons terminaux

Winter growth of the terminal buds 
L'élongation précoce de ces entre-nœuds est-elle particulière à cette année 1979 ? La comparaison de la figure 7 avec les figures 3 et 4 le suggère. En effet, il n'apparaît pas de différence entre la taille des bourgeons des clones polycycliques et monocycliques au début de la saison de végétation 1979 ( ${ }^{\text {er }}$ mars) (fig. 3 et 4 ), alors qu'elle est évidente sur la figure 7 et sur le tableau 1 au début de la saison de végétation 1980 (1 ${ }^{\text {er }}$ février). Il n'est pas exclu qu'entre ces deux dates, pour autant qu'elles soient comparables à des années différentes, les bourgeons des clones polycycliques se soient allongés. En effet, la formation des ébauches foliaires dans le second cycle est assez tardive dans l'hiver. Et cette formation s'accompagne généralement d'une élongation du bourgeon.

\section{2. - Décomposition de l'accroissement annuel}

\subsection{1. - Accroissements des différentes parties de la pousse annuelle}

Dans le tableau 1, figurent les longueurs des différentes parties de la pousse terminale de l'année 1979. Les mesures ont été faites après leur élongation, au cours de l'hiver 1979-1980.

- La pousse primaire des polycycliques est toujours inférieure et de manière significative à la pousse homologue (et donc totale) des monocycliques. Ce résultat est général et confirme ceux obtenus précédemment (KREMER, 1981). Cette supériorité des monocycliques pour cette pousse est due en partie à l'accroissement plus important de la partie feuillée par rapport aux polycycliques.

\section{TableaU 1}

Accroissement des différentes parties d'une pousse annuelle (en $\mathrm{cm}$ ) Height increment of different parts of an annual shoot (in centimeters)

\begin{tabular}{|c|c|c|c|}
\hline & Monocycliques & Polycycliques & Test $\mathrm{t}$ \\
\hline Partie stérile $\operatorname{PS}_{1} \ldots \ldots \ldots \ldots$ & 6,75 & 5,83 & $1,41 \mathrm{NS}$ \\
\hline Partie fertile $P_{1} \ldots \ldots \ldots \ldots$ & 57,18 & 51,94 & $2,59 *$ \\
\hline Pousse primaire $\ldots \ldots \ldots \ldots \ldots$ & 63,92 & 57,77 & $2,73 *$ \\
\hline Partie stérile PS $_{2} \ldots \ldots \ldots \ldots$ & & 5,61 & \\
\hline Partie fertile $\mathbf{P F}_{2} \ldots \ldots \ldots \ldots$ & & 2,29 & \\
\hline Pousse totale $\ldots \ldots \ldots \ldots \ldots$ & 63,92 & 65,68 & $0,76 \mathrm{NS}$ \\
\hline Bourgeon terminal .... & 14,64 & 10,83 & $5,41^{* *}$ \\
\hline
\end{tabular}

Test significatif :

* Au seuil de 5 p. 100

*** Au seuil de 1 p. 100. 
- La pousse secondaire représente 14 p. 100 de l'accroissement total chez les clones polycycliques. La partie «stérile» occupe la place la plus importante sur la seconde pousse. Ce n'est cependant pas le cas général : dans d'autres observations sur des arbres d’âge varié, la part de la partie feuillée dans la seconde pousse est aussi importante sinon plus que la partie «stérile». Enfin, il n'y a jamais d'écailles brunes à la base de la partie stérile de la seconde pousse.

- La pousse totale des polycycliques n'est pas significativement différente de celle des monocycliques. Dans d'autres exemples, où des observations pluri-annuelles avaient été faites, ce cas se présentait à l'occasion de certaines années sans doute plus sèches (KrEmer, 1981). Par contre, dans le cas le plus général, les polycycliques ont un accroissement supérieur à celui des monocycliques (KREMER, 1981).

- Le bourgeon terminal des monocycliques a une longueur plus importante que celui des polycycliques. Ce résultat est à rapprocher des courbes des figures 6 et 7 , qui montraient que le bourgeon des monocycliques s'allongeait de manière très nette durant la période estivale et automnale avant son entrée en dormance.

\subsection{2. - Allongement des entre-nouds à différents niveaux de la pousse annuelle (tableau 2)}

Que ce soit chez les polycycliques ou chez les monocycliques, il existe un gradient de l'allongement du bas de la pousse annuelle jusqu'au sommet, du moins en ce qui concerne la pousse primaire (PF 1). Ce gradient est à mettre en parallèle avec l'amélioration des conditions climatiques de début avril à fin juin. En effet, d'autres observations ont montré que ce sont tout d'abord les entre-nœuds à la base qui s'allongent. Quand cette élongation à la base s'est produite, il n'y a plus d'évolution par la suite à ce niveau. Seule la partie supérieure évoluera encore. Sauf au sommet de la partie feuillée de la pousse primaire, l'allongement des entre-nœuds des monocycliques est plus important que celui des polycycliques et plus particulièrement au milieu de la tíge. Cependant cette différence n'est significative que pour l'allongement moyen des entre-nœuds exprimé comme le rapport de la longueur de la partie feuillée (PF 1) sur le nombre total de brachyblastes présents sur cette partie. Sans doute l'élongation précoce des entre-nœuds, observée sur les bourgeons des monocycliques durant l'été précédent la saison de végétation affecte-t-elle non seulement la partie stérile (PS 1) mais aussi la partie feuillée (PF 1). Le gradient dallongement de la base au sommet de la pousse annuelle est rompu au niveau de la pousse secondaire pour les polycycliques. L'allongement des entre-nœuds passe de 2,03 à 1,45 mm. Pour Debazac (1963), c'est le cas le plus général et les allongements des entre-nœuds au niveau du sommet des parties feuiliées sont à mettre en relation avec le début de la sécheresse estivale.

\subsection{3. - Dénombrement des articles sur la pousse annuelle (tableau 3)}

Globalement sur l'ensemble de la pousse annuelle, les clones polycycliques forment deux fois plus de branches et de cônes et 18 p. 100 d'aiguilles et d'écailles en plus par rapport aux clones monocycliques.

Le supplément de branches, d'aiguilles et d'écailles provient exclusivement de la seconde pousse. En effet, au niveau de la première les clones des deux catégories 
ont le même nombre d'aiguilles et de branches. Par contre, les cônes qui ne se trouvent qu'au niveau du pseudo-verticille intermédiaire chez les clones polycycliques sont toujours en nombre supérieur chez ces derniers.

\section{Tableau 2}

Allongement des entre-ncuds sur différentes parties de la tige (en $\mathrm{mm}$ )

Length of internodes at different levels in an annual shoot (in millimeters)

\begin{tabular}{l|c|c|c}
\hline \hline & Monocycliques & Polycycliques & Test $\mathrm{t}$ \\
\hline Allongement à la base de $\mathrm{PF}_{1} \ldots \ldots \ldots$ & 1,22 & 1,18 & $0,46 \mathrm{NS}$ \\
\hline Allongement au milieu de $\mathrm{PF}_{\mathrm{t}} \ldots \ldots$ & 1,83 & 1,72 & $1,10 \mathrm{NS}$ \\
\hline Allongement au sommet de $\mathrm{PF}_{1} \ldots \ldots$ & 2,00 & 2,03 & $0,28 \mathrm{NS}$ \\
\hline Allongement moyens $/ \mathrm{PF}_{1} \ldots \ldots \ldots \ldots$ & 1,82 & 1,65 & $2,02 *$ \\
\hline Allongement sur la pousse secondaire $\ldots$ & & 1,45 & \\
\hline
\end{tabular}

\section{Tableau 3}

Dénombrement des articles présents sur la pousse annuelle

Number of steril bracts, dwatf shoots, female cones, and long shoots formed on an annual shoot

\begin{tabular}{|c|c|c|c|}
\hline & Monocycliques & Polycycliques & Test $t$ \\
\hline Nombre de brachyblastes sur $\mathrm{PF}_{1} \ldots \ldots$ & 306,59 & 306,76 & $0,01 \mathrm{NS}$ \\
\hline Nombre de cônes $\ldots \ldots \ldots \ldots \ldots \ldots$ & 1,54 & 3,0 & $3,92^{* * *}$ \\
\hline $\begin{array}{l}\text { Nombre de branches au pseudo verti- } \\
\text { cille intermédiaire } \ldots \ldots \ldots \ldots \ldots \ldots\end{array}$ & 3,76 & 3,52 & $1,03 \mathrm{NS}$ \\
\hline Nombre d'écailles sur PS $_{.} \ldots \ldots \ldots \ldots$ & & 41,86 & \\
\hline Nombre de brachyblastes sur $\mathrm{PF}_{2} \ldots \ldots$ & & 14,48 & \\
\hline $\begin{array}{c}\text { Nombre de branches au second pseudo } \\
\text { verticille } \quad \ldots \ldots \ldots \ldots \ldots \ldots \ldots\end{array}$ & & 5,03 & \\
\hline Nombre total d'articles $* \ldots \ldots \ldots \ldots$ & 311,90 & 374,69 & $5,46^{* *}$ \\
\hline
\end{tabular}

* Ce nombre total ne tient pas compte des écailles «stériles» de PF1.

Nous n'avons jamais observé comme Debazac (1963) de fructification femelle au niveau du second pseudo-verticille chez les clones polycycliques. Il est vrai que 
ses observations avaient été faites dans les Maures, dans des conditions écologiques différentes.

Même en ne tenant pas compte du pseudo-verticille intermédiaire, dont les branches sont toujours plus fines, les clones polycycliques produisent nettement plus de branches. La conséquence possible est un développement plus important de la surface foliaire qui par ailleurs est déjà augmentée au niveau d'une branche par la production d'une seconde pousse. Cependant, nous n'avons pas d'estimation chiffrée de ces différences de surface foliaire.

\section{3. - Corrélations entre composantes de la croissance en hauteur (tableaux 4 et 5)}

Ces corrélations ont été estimées à l'intérieur de chaque population des clones monocycliques et polycycliques. Par ailleurs, le nombre de copies par clone étant très faible et aucune répétition n'ayant été faite au niveau de la plantation, l'estimation a été faite au niveau phénotypique.

\subsection{1. - Liaisons entre composantes}

L'allongement des premiers entre-nœuds est lié au nombre total d'entre-nœuds présents dans la tige embryonnaire, que ce soit pour les clones polycycliques ou monocycliques (tableau 4). Plus ce nombre est élevé, et moins l'allongement sera important (et inversement) : cette corrélation négative diminue à mesure que l'on se situe à un niveau plus élevé sur la pousse annuelle. Il y a donc une certaine compensation entre ces deux composantes de la croissance, qui se réalise surtout au niveau des premiers entre-nœuds. Autrement dit, durant la période d'élongation de la tige, le nombre d'entre-nœuds qui reste à allonger tend à s'uniformiser entre les différents individus au cours du temps.

Les corrélations négatives relativement fortes entre le nombre total d'entrenœuds et leur allongement moyen sur l'ensemble de la partie feuillée de la pousse primaire peuvent être dues en partie à des auto-corrélations. En effet, soit $n$ le premier caractère, le second s'exprime $X / n$ et, par définition même, une variation du premier entraîne une variation inverse du second.

\subsection{2. - Liaisons entre composantes et accroissement de PFI}

La différence essentielle entre clones polycycliques et monocycliques naît des corrélations entre l'accroissement total réalisé et les composantes de celui-ci (tableau 5).

- Chez les clones monocycliques, la longueur de la partie feuillée de la pousse primaire est avant tout liée à l'allongement des entre-nœuds. Cette liaison est d'autant plus étroite, que le niveau de l'allongement se situe au milieu ou au sommet de la partie feuillée. Par ailleurs, la longueur de cette dernière est pratiquement indépendante du nombre d'entre-nœuds.

- Chez les clones polycycliques, l'allongement des entre-nouds au niveau du sommet de la partie feuillée explique une grande partie de l'accroissement total de cette partie, qui, par ailleurs, est pratiquement indépendant de l'allongement des entrenœuds à la base et au milieu. En revanche, la longueur de la partie feuillée est corrélée au nombre d'entre-nœuds. 


\section{IV. - Discussion}

\section{1. - Constitution de la pousse annuelle}

Lélaboration de la pousse annuelle du pin maritime a été étudiée en détail par Morisset (1966) au moins en ce qui concerne l'initiation des différents articles constituant la pousse annuelle. Ses observations complétées par les nôtres sont résumées dans la figure 8, qui représente le déroulement dans le temps des différentes phases du développement végétatif aboutissant à la constitution de la pousse annuelle.

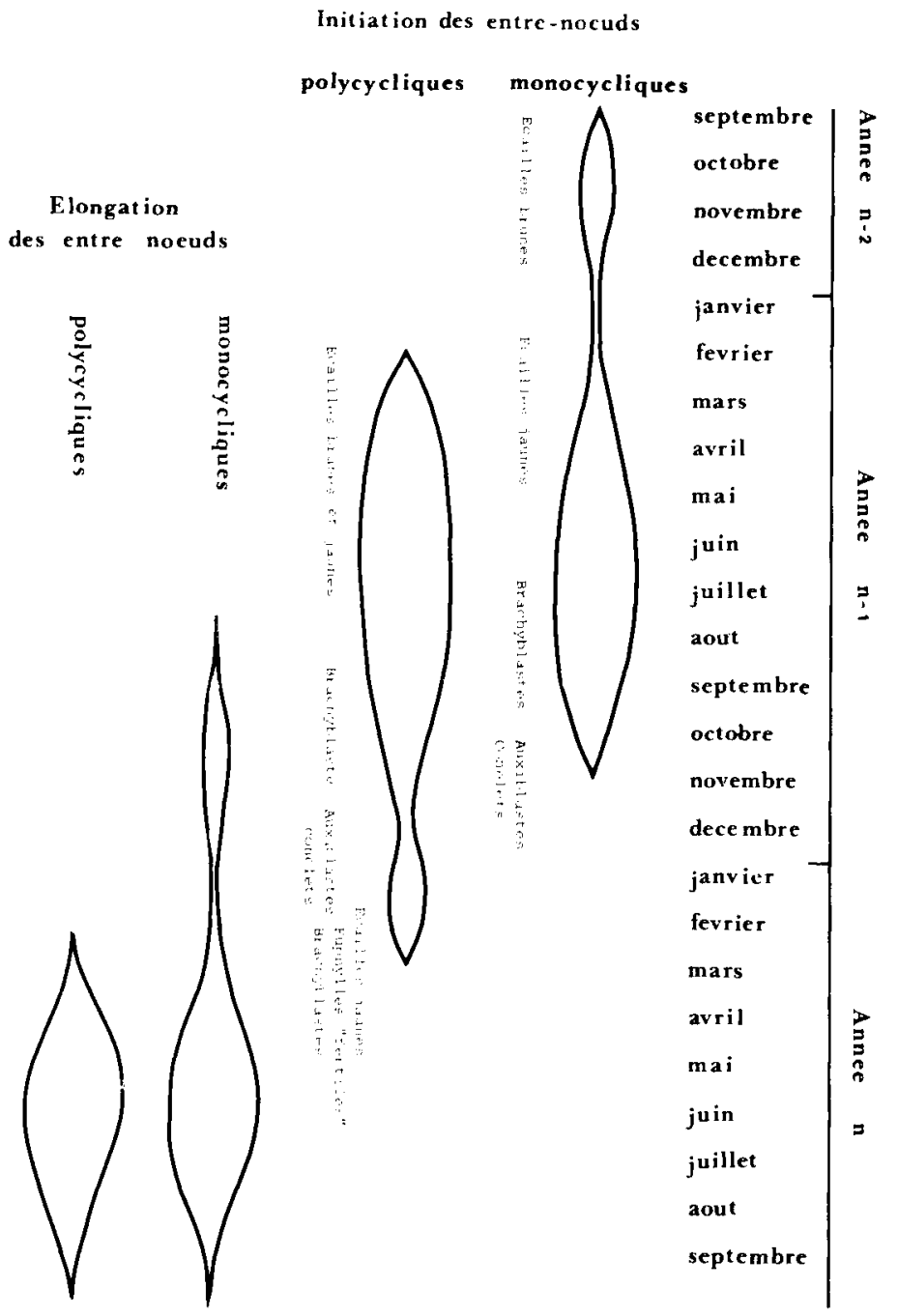

FIG. 8

Chronologie de la constitution de la pousse de lannée $n$ Chronology of the annual shoot formation 
Cette figure est une représentation schématique et sans doute caricaturale, qu'il faudra interpréter avec prudence en tenant compte des conditions écologiques de chaque année.

\subsection{1. - Initiation des euphylles et développement des structures axillaires}

D’après Morısset (1966), au niveau de linitiation, la différence essentielle entre pousse bicyclique et pousse monocyclique est liée à la durée de fonctionnement de lanneau initial. En effet, les articles de la pousse primaire des individus polycycliques et ceux de la pousse totale des individus monocycliques sont initiés durant la même période (mai à septembre). En revanche, alors que l'anneau initial des premiers continue à fonctionner et à initier les articles de la pousse secondaire, les bourgeons des seconds entrent en dormance durant l'automne. Les dénombrements des articles sur la pousse annuelle tendent à confirmer ces observations morphogénétiques de MoRISSET : le nombre de brachyblastes présents sur la première pousse chez les polycycliques est équivalent à celui présent sur la pousse homologue des monocycliques, à l'exception des cônelets. Il apparaît done que linitiation de ces derniers soit plus tardive et appartienne déjà, au plan morphogénétique, à la formation de la seconde pousse. Pour Morisset, les ébauches des cônelets femelles sont repérables à partir de la fin du mois d'octobre sur les arbres monocycliques. Mais les auxiblastes et les cônelets du pseudo-verticille terminal des monocycliques n'évoluent pas pendant l'hiver, alors que ceux du pseudoverticille intermédiaire des polycycliques se développent en même temps que l'élaboration de la pousse secondaire. Le ralentissement ou le non développement des ébauches des cônelets chez les clones monocycliques peut éventuellement entraîner un taux d'avortement plus important chez ces derniers.

Parmi les espèces suivant le même schéma morphogénétique, c'est-à-dire la formation des deux pousses durant l'année n-1 et l'élongation lannée n, c'est Pinus contorla qui se rapproche le plus du pin maritime. L'expression de la seconde pousse peut être occasionnelle tout comme chez le pin maritime. Globalement, les pins polycycliques de cette espèce ont une période d'initiation d'euphylles plus longue que les monocycliques (Owens \& Molder, 1975). Alors que chez le pin maritime cette durée supplémentaire d'activité mitotique est de plusieurs mois, chez Pinus contorta elle n'est que de quelques semaines. Aucun dénombrement de brachyblastes ne montre cependant si elle correspond à un supplément d'euphylles initićs. Par ailleurs, si VAN DEN BERG \& LANNER (1971) dune part et OWINS \& MOLDER (1975) d'autre part mettent en évidence le développement plus précoce des auxiblastes et cônelets du pseudo-verticille intermédiaire par rapport à ceux du pseudo-verticille terminal des monocycliques, ils ne donnent pas de résultats chiffrés de différences de fructification entre arbres polycycliques et monocycliques.

Enfin, il apparaît qu'il y a une relation très étroite entre développement végétatif et développement des organes reproductifs chez Pinus taeda (GreENwood, 1980). Chez cette espèce, la pousse annuelle est issue d'un ou de deux cycles préformés, auxquels sajoutent un ou deux autres non préformés. Greenwood observe que les rameaux monocycliques n'ont jamais de fructification femelle. Par ailleurs, en ne considérant que les rameaux toujours polycycliques à deux cycles préformés, les clones dont le second cycle préformé est particulièrement important ont tendance à produire moins de cônelets. Pour cet auteur, les différences d'abondance de fructification entre clones ne sont pas liées à des différences de comportements phénolo- 
giques (temps, durée de la différenciation des organes reproductifs en relation avec les facteurs du milieu) mais à des différences de développement végúlatif (vigueur) entre clones. Selon ses propres termes "l’activité méristématique responsable de linitiation des ébauches foliaires (dont dentre-nouds) peut inhiber la différenciation des ébauches florales».

En essayant de traduire ces résultats au niveau du pin maritime cela voudrait dire qu'à l'intérieur de la population polycyclique, il y a une corrélation négative entre abondance de fructification et longueur du second cycle. Le nombre de copies par clone étant trop faible, nous n'avons pas pu estimer ce coefficient de corrélation au niveau moyenne clonale.

\subsection{2. - Elongation des entre-nouds}

Si les clones polycycliques et monocycliques peuvent être différenciés par la période d'activité des méristèmes apicaux responsables de l'initiation des euphylles, il en va de même pour la période de fonctionnement des méristèmes subapicaux et médullaires responsables de l'élongation des entre-nœuds. En effet, les bourgeons des clones monocycliques s'allongent durant l'été et l'automne précédent l'année où l'élongation de la tige se réalise. Cette élongation précoce se traduit à la fois au niveau de la partie stérile et de la partie feuillée. Cependant, toutes choses étant égales par ailleurs, c'est-à-dire pour un même nombre d'entre-nœuds, la différence entre longueur de la pousse totale des monocycliques et celle de la pousse primaire des polycycliques est telle que la précocité de l'élongation du bourgeon chez les premiers n explique pas totalement leur supériorité sur les seconds. Autrement dit, pendant la période d'élongation maximale de la mi-avril à la mi-juin, les arbres monocycliques allongent plus leurs entre-nouds que les arbres polycycliques.

Il n’apparaît pas de différence pour le début et la fin de la croissance pendant la période printanière où son intensité est maximale entre les clones polycycliques et les clones monocycliques. En particulier, en ce qui concerne les premiers, la transition entre élongation des derniers entre-nœuds de la première pousse et les premiers de la seconde se fait de manière continue, sans rupture dans la courbe de croissance même si les entre-nœuds de la seconde pousse s'allongent globalement moins que les derniers de la première pousse des monocycliques.

Ces observations vont dans le même sens que celle d'Illy \& Castaing (1966) qui notaient qu’il «napparaissait pas de liaison entre la formation de verticille et la croissance en hauteur».

Autant pour linitiation des euphylles que pour l'élongation des entre-nouds (voir figure 7), il faut préciser qu il s'agit d’un comportement adulte tel quil a pu être observé sur des arbres de douze ans dans le cas de MoRISSET et sur des greffes ayant 15 années, dont les greffons ont été prélevés sur des arbres de quarante à soixante ans. Le comportement juvénile se caractérise par une croissance «libre 》 au sens de LANNER ; il y a initiation d'euphylles et les entre-nœuds correspondant s'allongent la même année. C"est ainsi que sur des arbres plus jeunes (Illy \& Castaing, 1966) un second pic de croissance peut être observé au mois de juillet et août, et on peut observer notamment dans le cas de fertilisation, 3 cycles morphogénétiques sur un accroissement annuel. Il n'est pas exclu que dans le cas darbre adulte, la fertilisation appliquée au printemps se traduise par un retour à une croissance de type "libre»: 
cela expliquerait les fréquences plus élevées de seconds cycles en cas de fertilisation observées par MAugE et al. (1970), et les moins bonnes corrélations entre les accroissements annuels (correspondant aux années avec fertilisation) et les accroissements d'années ultérieures.

En tout cas, nous avons pu observer sur les bourgeons prélevés au mois de février sur des arbres de onze ans de provenances différentes, les seconds cycles préformés, dont les euphylles et certaines ébauches foliaires sont nettement distingables (données non publiées).

\section{2. - Dynamique de l'allongement des entre-nouds}

De récentes observations sur du matériel différent montrent qu'il existe un centre de croissance qui se déplace le long de la tige durant son élongation. Pour une position donnée du centre, il y a un gradient d'élongation du bas vers le haut. Ainsi au début de la saison de végétation, l'ensemble de la tige embryonnaire s'allonge, mais les entre-nœuds à la base s'allongent plus rapidement que ceux du sommet. Quand les premiers ont atteint le maximum de leur élongation, ils n'évoluent plus et seuls les entre-nœuds supérieurs, tout en respectant le gradient, s'allongent. Le centre de croissance s'est ainsi déplacé. Il continue de se déplacer ainsi tout au long de la saison de végétation.

Dans de telles conditions, les entre-nœuds à la base et au sommet de la pousse ne s'allongent respectivement qu'en début et en fin de saison de végétation. Par contre ceux du milieu s'allongent pendant une période plus longue. On comprend ainsi aisément les meilleures corrélations entre longueur de la partie feuillée et allongement des entre-nœuds au milieu de PF 1 (tableau 5) pour les clones monocycliques et entre longueur de PF 1 et allongement des entre-nœuds au milieu et au sommet de PF 1 pour les clones polycycliques. Ce schéma du développement télescopique de la pousse permet également d'interpréter le faible allongement des entre-nœuds à la base de PF 1 (tableau 2). S'allongeant uniquement en début de la saison de végétation, ils souffrent des températures peu élevées qui règnent à cette époque.

\section{Tableau 4}

Coefficients de corrélation phénotypique entre nombre d'entre-nutuds sur la partie feuillée de la première pousse $\left(P F_{3}\right)$ et leur allongement à différents niveaux sur cette partie

Phenotypic correlation coefficient values between number of internodes on the dwarf shoot segment of the first cycle and the internode length at different levels in that segment

\begin{tabular}{|c|c|c|c|c|}
\hline & $\begin{array}{l}\text { Allongement } \\
\text { à la base }\end{array}$ & $\begin{array}{l}\text { Allongement } \\
\text { au milieu }\end{array}$ & $\begin{array}{l}\text { Allongement } \\
\text { au sommet }\end{array}$ & $\begin{array}{l}\text { Allongement } \\
\text { moyen sur } \mathbf{P F}_{2}\end{array}$ \\
\hline Monocycliques $\ldots \ldots$ & $-0,57^{* * *}$ & 一 $0.46^{*}$ & $-0,27$ & $-0,70^{* *}$ \\
\hline Polycycliques ....... & $--0,60^{* * *}$ & $-0,38^{*}$ & $-0,13$ & $-0,58 * *$ \\
\hline \multicolumn{5}{|c|}{$\begin{array}{l}\text { Coefficient de corrélation: } \\
* \quad \text { Significatif au seuil de } 5 \text { p. } 100\end{array}$} \\
\hline
\end{tabular}


Si l'allongement d'un entre-nœud donné dépend de sa position dans la tige, il dépend aussi et de manière très étroite du nombre d'entre-nœuds qu'il reste à allonger. Ce résultat est traduit par les corrélations négatives entre le nombre d'entre-nœuds présents sur la tige et leur allongement à différents niveaux (voir tableau 4). Nettement significatives quand l'allongement à la base de la partie feuillée est pris en compte, elles le demeureraient sans doute aux autres niveaux si le second caractère était le nombre d'entre-nœuds restant à allonger au lieu du nombre total initial. Tout se passe comme si la vitesse de déplacement du centre de croissance était plus élevée pour les arbres dont il reste encore de nombreux entre-nœuds à allonger, alors qu'elle est nettement plus faible pour ceux n'ayant qu'un faible nombre. Les résultats du tableau 4 permettent d'affirmer que ce raisonnement peut s'appliquer à la fois aux monocycliques et aux polycycliques. Enfin, la durée d'élongation étant la même pour les deux catégories, la vitesse de déplacement du centre de croissance doit être plus rapide chez les polycycliques.

TABLEaU 5

Coefficients de corrélation phénotypique éntre longueur de la partié feuillée de la première pousse $\left(P F_{1}\right)$ et différentes composantes de cette partie

Phenotypic correlation coefficient values between the length of the dwarf shoot segment of the first cycle and different components of that segment

\begin{tabular}{c|c|c|c|c|c}
\hline & $\begin{array}{c}\text { Nombre } \\
\text { d'entre- } \\
\text { nouds }\end{array}$ & $\begin{array}{c}\text { Allongement } \\
\text { à la base }\end{array}$ & $\begin{array}{c}\text { Allongement } \\
\text { au milieu }\end{array}$ & $\begin{array}{c}\text { Allongement } \\
\text { all sommet }\end{array}$ & $\begin{array}{c}\text { Allongement } \\
\text { moyen } \\
\text { sur PF }\end{array}$ \\
\hline Monocycliques ..... & $-0,02$ & 0,25 & $0,76^{* *}$ & $0,73^{* * *}$ & $0,77^{* *}$ \\
\hline Polycycliques ...... & $0,40^{*}$ & $\simeq 0$ & 0,27 & $0,74^{* *}$ & $0,50^{* * *}$ \\
\hline
\end{tabular}

Si le processus délongation des entre-nœuds est identique chez les pins monocycliques et polycycliques, le résultat in fine diffère quelque peu. Pour les premiers laccroissement de la partie feuillée est surtout lié à l'allongement moyen des entre-nœuds. Pour les seconds, si cette liaison existe, elle existe également avec le nombre d'entre-nœuds. La corrélation entre le nombre d'entre-nœuds et l'accroissement de la partie feuillée (PF 1) des polycycliques est beaucoup plus nette chez d'autres espèces (Pinus palustris, Allen \& SCARbrough, 1970). Il faut préciser que la variation du nombre d'entre-nœuds était nettement plus importante (200 à 600) alors qu'elle n'est que de 270 à 400 dans notre exemple. Vérifiée sur deux ans, la corrélation était d'autant plus élevée que les conditions écologiques sévissant durant la période d'élongation étaient favorables. Cela pourrait être une explication a posteriori de nos résultats. En tout état de cause, les mesures devraient être répétées sur plusieurs années. Enfin, l'initiation des brachyblastes, tout en contribuant à la constitution d'une pousse nouvelle, participe également à l'élaboration de la surface foliaire. Sans doute les deux fonctions ne sont pas indépendantes et les résultats obtenus par les comptages d'aiguilles ne doivent pas exclusivement être interprétés sous l'angle de la croissance en hauteur. 


\section{V. - Conclusion}

Au plan du fonctionnement méristématique, il apparaît donc une distinction très nette entre clones monocycliques et polycycliques. La durée de l'activité mitotique des méristèmes apicaux des clones polycycliques est nettement plus longue que celle des monocycliques. A l'inverse, la durée d'activité mitotique des méristèmes subapicaux est nettement plus longue pour les clones monocycliques que pour les clones polycycliques. Dès lors, se posent les questions du déterminisme du déclenchement, du fonctionnement et de l'arrêt de l'activité de ces méristèmes. L'alternative offerte (polycyclique ou monocyclique) semble montrer au moins qu'il n'y a pas indépendance entre le fonctionnement des deux catégories de méristèmes. Si l'alternative est exclusive, le monocyclisme est donc synonyme de non-polycyclisme, et l'élongation des bourgeons durant l'été inhibe donc la formation d'une seconde pousse l'hiver. En tout état de cause, cette étude mériterait d'être poursuivie à un niveau plus fin : fonctionnement de l'anneau initial en ce qui concerne l'initiation des euphylles, division et croissance des cellules au niveau des entre-nœuds pour leur élongation. Enfin, si un tel prolongement est envisagé, il s'avère à la lumière de nos observations que des relevés pluriannuels devraient être effectués. Certains indices, dans notre propre étude limitée à des observations sur une seule saison de végétation, ne permettent pas de généraliser nos résultats. Cette étude s'attachant en partie à suivre la croissance durant une année donnée, est inévitablement liée aux conditions écologiques régnant durant cette saison de végétation.

$\mathrm{Au}$ plan du déterminisme génétique de la croissance en hauteur, il serait très intéressant d'estimer les paramètres génétiques des différentes composantes de la croissance. Au plan pratique et au niveau de l'amélioration génétique du pin maritime, l'hybridation entre arbres monocycliques et arbres polycycliques pourrait aboutir à un effet d'hétérosis dû à une complémentarité entre les deux composantes que les deux populations présentent au niveau optimal.

Reçu pour publication en avril 1981.

\section{Summary}

\section{Components of height growth in maritime Pine (Pinus pinaster Ait.)}

Nodes were counted on an annual shoot increment. Growth curves of shoot elongation were constructed. These results and morphogenetic studies of bud development by MoRIsSET (1966) lead to description of different steps in initiation and development of annual shoot of maritime pine. There are differences between polycyclic and monocyclic clones. Cataphylls of the second morphogenetic cycle of the polycyclic trees are initiated during winter time while apical meristems of monocyclic trees are dormant during this period.

Buds of the monocyclic trees begin to elongate during summer before the year of growth. Polycyclic trees have more sheath scales, cones and branches but their first shoot length is less than the total shoot length of monocyclic trees. Internode elongation of the last ones is more important. This is due to the earlier elongation and to a higher growth rate during spring.

Within the monocyclic population, annual shoot increment is mainly explained by internode elongation at different levels. Within the polycyclic population, both number of internodes and their elongation explain first annual shoot increment.

Key words : Morphogenesis, height growth, cataphyll initiation, stem unit elongation, polycyclism, Pinus pinaster. 


\section{Références bibliographiques}

Al.len R.M., Scarbrough N.M.. 1970. Morphology and length correlated in terminal flushes of longleaf saplings. U.S.D.A. Forest Service Research Paper, S0-53, 15 p.

Camefort H., 1956. Variations phyllotaxiques chez quelques gymnospermes. Ann. Sci. nat., Biol. vég., 11, 17, 85-185.

Cannell M.G.R., Thompson S., Lines R., 1976. An analysis of inherent differences in shoot growth within some north temperate conifers. In : Tree Physiology and Yield Improvement, 173-205, Eds M.G.R. Cannell et F.T. Last, Academic Press, New York.

Cannell M.G.R., 1978. Components of conifer shoot growth. In : Proceedings of the fifth North American forest biology workshop, 313-318, Eds C.A. Hollis and A.E. Squillace, University of Florida, Gainesville, Florida.

Cannell M.G.R., Bowler K.C., 1978. Phyllotactic arrangements of needles on elongating conifer shoots : a computer simulation. Can. J. For. Res., 8, 138-141.

Castaing J.Ph., Illy G., 1966. Rythme saisonnier de croissance en diamètre et en hauteur chez le pin maritime. Bull. Soc. bot. Fr., 114, 173-179.

Debazac E.F., 1963. Morphologie et sexualité chez les pins. Rev. for. fr., 14, 4, 213-303.

Doak C.C., 1935. Evolution of foliar types, dwarf shoots and cone scales of Pinus. IIl. Biol. Monogr., 13, 1-106.

Greenwood M.S., 1980. Reproductive development in Loblolly Pine. I. The early development of male and female strobili in relation to the long shoot growth behaviour. Am. J. Bot., 67, 10, 1414-1422.

Kremer A., 1981. Déterminisme génétique de la croissance en hauteur du Pin maritime (Pinus pinaster Ait.). I - Rôle du polycyclisme. Amn. Sci. for., 38, 2, 199-222.

Lanner R., 1976. Patterns of shoot development in Pinus. In : Tree Physiology and Yield Improvement, 233-243, Eds M.G.R. Cannell et F.T. Last, Academic Press. New York.

LANNER R., 1978. Development of the terminal bud and shoot of slash pine saplings. For. Sci, 24, 2, 167-179.

Mauge J.M., Alazar P., Castaing; J.Ph., Levadou D., 1976. Critères de sélection pour la croissance en hauteur du pin maritime. Ann. Rech. sylvic., A.F.O.C.E.L., 331-350.

Morisser R., 1966. Etude morphogénétique du bourgeon terminal de pin maritime. D.E.S. de Sciences naturelles, Université de Bordeaux, 39 pages.

OWens J.N., Molder M., 1975. Development of long shoot terminal buds of Pinus contorta ssp contorta. In : Proceedings of management of lodgepole pine ecosystems symposium. 86-103. Ed. D.M. Baumgartner, Washington State University, Pullman, Washington.

Pollard D.F.W., Logan K.T., 1976. Inherent variation in «free " growth in relation to numbers of needles produced by provenances of Picea mariana. In : Tree physiology and yield improvement, 245-252, Eds. M.G.R. Cannell et F.T. Last, Academic Press, New York.

Pollard D.F.W., Logan K.T., 1977. The effects of light intensity, photoperiod, soil moisture potential and temperature on bud morphogenesis in Picea species. Can. J. For. Res.. 7. 2, 415-421.

Van den Berg D.A., Lanner R.M., 1976. Bud development in Lodgepole pine. For. Sci., 17, 479-486. 